\title{
Defect-Photonic Crystal Fiber structure of Zero Chromatic Dispersion and very low
} Confinement loss

Patrick Atsu Agbemabiese' ${ }^{1}$ Jay Denton Agamah ${ }^{2}$, Jacob Ayehquaye Nartey ${ }^{3}$ and Moses K. Torkudzor ${ }^{4}$

1, 2, 3,4 Department of Electrical/Electronic Engineering, Koforidua Technical University. Mailing address: Koforidua Technical University, P.O. Box KF 981, Koforidua, Ghana.

${ }^{1}$ Correspondence: Paagbemabiese@gmail.com, Tel: +233244180111

\section{Abstract}

Photonic crystal fibers are being designed with promising results owing to design flexibility and structure geometry. A four-ring structure with ring defects has been proposed. The results show that Photonic crystal fiber with the third ring removed has very low confinement loss of $1.17 \times 10^{-4} \mathrm{~dB} / \mathrm{km}$ at $1.55 \mu \mathrm{m}$, chromatic dispersion of $69 \mathrm{ps} / \mathrm{km} . \mathrm{nm}$ at $0.75 \mu \mathrm{m}$ and zero chromatic dispersion at $0.86 \mu \mathrm{m}$. The results also show that removing only the third ring reduces chromatic dispersion at shorter wavelengths than at longer wavelengths. Vectorial Finite element method is used for this work. The proposed fiber can be used for short and medium transmission applications.

Keywords: chromatic dispersion, defects, photonic crystal fibers, confinement loss, perfectly matched layer

Citation: Agbemabiese, P. A., Agamah, J. D., Nartey, J. A. and Torkudzor, M. K. (2021). Defect-Photonic Crystal Fiber structure of Zero Chromatic Dispersion very low Confinement loss. International Journal of Technology and Management Research (IJTMR), Vol. 6 (2): Pp.96-105.

Received: March 20, 2021

Accepted: September 1, 2021 


\subsection{Introduction}

Photonic bandgap effect came to light from the works of Yablonovitch and John (Yablonovitch, 1987) in the study of spontaneous emission control and confinement of light in periodic materials (Bjarklev, Broeng, \& Bjarklev, 2012). Dielectric materials are arranged in a periodic form. If total photonic bandgap is considered, the frequencies obtained does not allow any propagation when the photonic crystal is infinite. Certain frequencies propagate, should a defect be introduced into the infinite photonic crystal. This defect could be a point defect which changes the periodicity in the crystal but allowing for light confinement in the area of the defect as the light comes from the defect. Another defect which also confines the light at the area of the defect and allow for propagation along the defect is the linear defect. Guiding light under the condition of linear defect can also occur in a two dimensional periodicity. Holes are inserted in a silica material to create a lower refractive index called the cladding as oppose to the core with high refractive index. Cladding which is holy, is made of two dimensionally periodic array of holes. Photonic crystal fiber (PCF) could be either holy core or of solid core type. The type that is widely used is the solid core due to its ease of fabrication. Defects can be done at the preform stage of fabrication with ease which makes creating defects in photonic crystal fiber of less fabrication difficulty.(d ric Zolla, 2005)

A paper by (Biswas et al., 2019) have proposed a circular air holes type of PCF and created a defect in the core by eliminating a single hole and has achieved a chromatic dispersion of $-1044 \mathrm{ps} / \mathrm{km}$.nm at a wavelength of $1.55 \mu \mathrm{m}$ but the work has not been extended to investigate confinement loss property. Rabiul Hasan et al (Hasan, Islam, Rifat, \& Hasan, 2017), increased birefringence by removing two holes in a circular air hole photonic crystal fiber structure to create artificial defects within the core. The results indicates an ultra-high birefringence of $2.64 \times 10^{-2}$ and negative dispersion values from $-242.22 \mathrm{ps} / \mathrm{km} . \mathrm{nm}$ to $-762.6 \mathrm{ps} / \mathrm{km} . \mathrm{nm}$ at a wavelength of $1.3 \mu \mathrm{m}$ to $1.67 \mu \mathrm{m}$. Further to this work, another research by Lee et al (Lee, Lee, Jung, Oh, \& Kim, 2016) implemented two line defects inside the core to obtain birefringence in the order of $10^{-2}$ with an improvement of dispersion from $400 \mathrm{ps} / \mathrm{km} . \mathrm{nm}$ at a wavelength of $1.26 \mu \mathrm{m}$ and a confinement loss of $10^{-3} \mathrm{~dB} / \mathrm{km}$. In another PCF structure, tiny holes have been introduced into the core to create a defect (Talukder, Isti, Nuzhat, \& Biswas, 2020). In all the research papers mentioned, the defects have been created in the core by adding or eliminating a hole. However, none shows the effect of a removal of one or two rings on the optical properties of the structure especially with all circular air holes PCF structure. Also some PCF structures with circular air holes have been replaced by elliptical holes in order to create some sort of defects in (Ghunawat, Chandra, \& Singh, 2017; Halder \& Hossain, 2016).

In this paper a four ring PCF structure with circular air holes and three conditions of ring defect has been designed to achieve a very low confinement loss of $1.17 \times 10^{-4} \mathrm{~dB} / \mathrm{km}$ at $1.55 \mu \mathrm{m}$, chromatic dispersion of $-69 \mathrm{ps} / \mathrm{km} . \mathrm{nm}$ at $0.75 \mu \mathrm{m}$ and zero chromatic dispersion at $0.96 \mu \mathrm{m}$ is proposed. Propagation properties of the proposed PCFs have been analyzed using full vectorial Finite Element Method

\subsection{Methodology}

2.1 Design methodology of proposed PCF structure

Proposed Hexagonal PCF structures; PCF1, PCF2 and PCF3's cross-sectional view are shown in figures 1.1, 1.2 and 1.3 respectively. The designs consist of a cladding of circular air holes and solid core. For the background material, silica is used, which is industrially available. The refractive index considered for the cladding is 1 . The 
proposed designs are progressive and consist of three air hole rings in PCF 1, two air hole rings in PCF 2 and PCF 3. A perfectly matched layer has been provided to absorb radiated waves with small reflections light. This design is made up of four rings. All the three PCF's have air filling fraction of $d / \wedge=0.6$. The rings are designed such that the pitch is kept at $2.3 \mu \mathrm{m}$ for all the PCF structures. In PCF1 the air hole radius for each hole in ring one is given by $1.035 \mu \mathrm{m}$ and ring two except ' $\mathrm{a}$ ' and 'b' is given by $0.8625 \mu \mathrm{m}$. The fourth ring have holes with radii of $0.5175 \mu \mathrm{m}$.

The PCF structures have been designed to study the effect of the defect created by the removal of some rings. In PCF1 the third ring has been removed. In PCF2 the first and the second ring have been removed. Also, in PCF3 the first, second and third ring has been replaced with one ring with a pitch of $4 \mu \mathrm{m}$. The hole labelled 'c' and 'd' are given by a radii of $1.725 \mu \mathrm{m}$ and $2.07 \mu \mathrm{m}$ respectively.

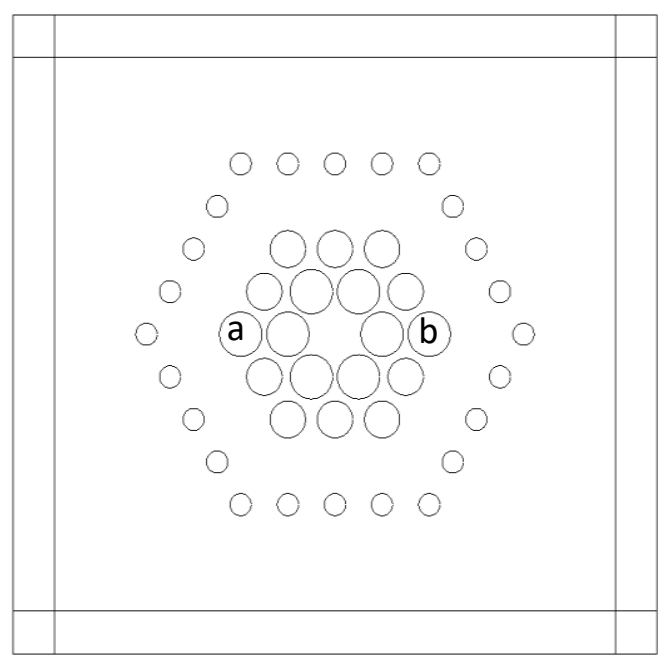

Figure 1.1: PCF 1 (third ring is removed)

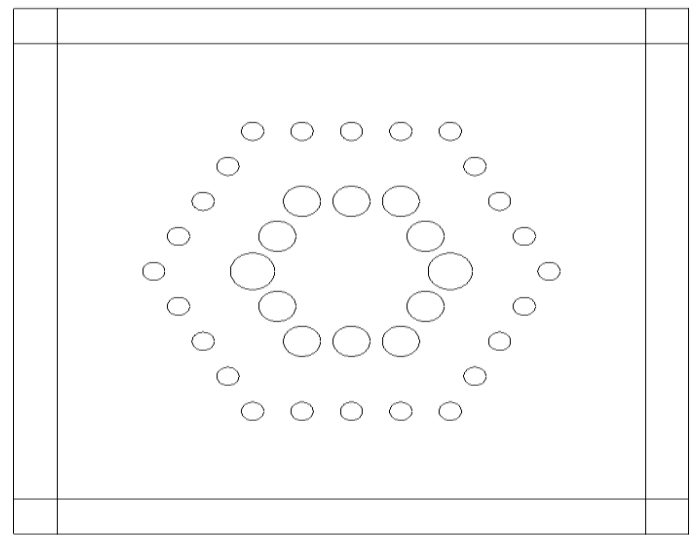

Figure 1.2: PCF 2, first and third ring removed 


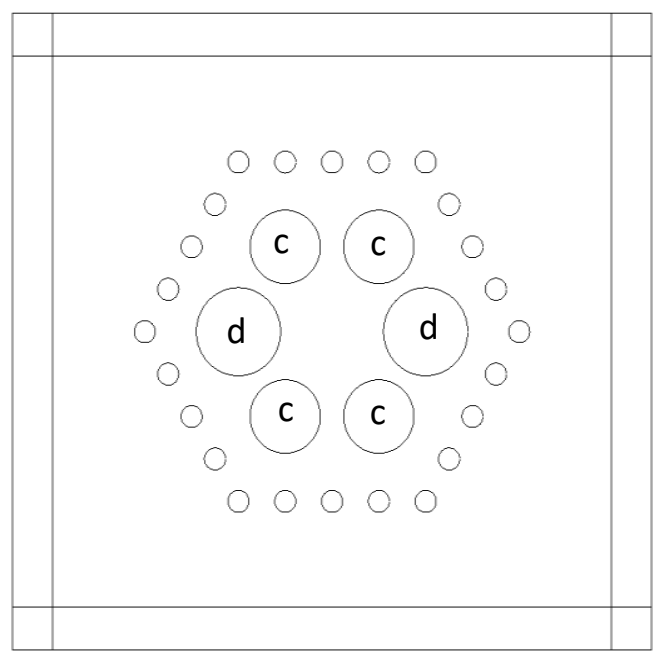

Figure 1.3: $P C F$ 3, 1st, 2nd and 3rd ring have been removed and replaced with one ring with bole-hole spacing of $4 \mu m$

\subsection{Numerical method}

The design was done using Comsol Multiphysics with perfectly matched layer (PML) boundary condition. Optical properties were investigated using results obtained from the simulation. The vectorial equation obtained from the Maxwell's curl equation which is solved by finite Element method is given by (Bjarklev et al., 2012) shown in equation 1;

$$
\nabla \times\left(\left[S^{-1}\right] \nabla \times E\right)-k_{0}^{2} n^{2}[S] E=0 \ldots \ldots \ldots \ldots \ldots . .1
$$

Where, $[\mathrm{s}]^{-1}$ is an inverse matrix of $[\mathrm{s}], \mathrm{E}$ is the electric field vector, $[\mathrm{s}]$ is the PML matrix, $\lambda$ is the wavelength, $\mathrm{k}_{0}$ $=2 \pi / \lambda$ gives the wave number in vacuum, $\mathrm{n}$ is the refractive index of the domain.

SellMeier equation used (Bjarklev et al., 2012) is shown in equation 2:

$$
n^{2}=1+\frac{\mathrm{B}_{1} \lambda^{2}}{\lambda^{2}-c_{1}}+\frac{\mathrm{B}_{2} \lambda^{2}}{\lambda^{2}-c_{2}}+\frac{\mathrm{B}_{3} \lambda^{2}}{\lambda^{2}-c_{3}} \ldots \ldots \ldots \ldots . . . .2
$$

Where $\mathrm{n}$ is the refractive index of the silica, $\lambda$ is the wavelength in $\mu \mathrm{m}, \mathrm{B}_{1,2,3}$ and $\mathrm{C}_{1,2,3}$ are sellMeier coefficients. The table 1 shows the sellmeier constants.

Table 1 Values of sellmeier coefficients for background silica material

\begin{tabular}{ll}
\hline Parameters & Constants \\
\hline B1 & 0.69675 \\
B2 & 0.408218 \\
B3 & 0.890815 \\
C1 & $4.67914826 \mathrm{e}-3$ \\
C2 & $1.35120631 \mathrm{e}-2$ \\
C3 & 97.9340025 \\
\hline
\end{tabular}




\subsection{The Chromatic Dispersion property}

Chromatic dispersion is the sum of wavelength dispersion and material dispersion. Wavelength dispersion is a parameter that depends on the diameter of the photonic crystal core and the difference realized between the refractive index of the air hole cladding and solid core. Material dispersion on the other hand relies on the refractive index of the material is created by the interaction between the electrons in the material and ions. Refractive index difference between the core and the cladding influences the chromatic dispersion. The spacing between holes and size of the air holes can be adjusted to help obtain very useful dispersion properties. The equation 3 is used for the computation of the chromatic dispersion.

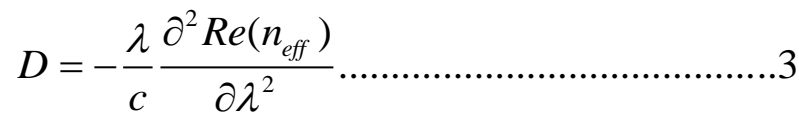

Where $\mathrm{c}$ is the velocity of light and $\lambda$ the operating wavelength

\subsection{Confinement Loss}

In PCF, the mode leak from the core to the finite holes in the cladding. This occurs in-between the holes. The confinement loss $\left(\mathrm{C}_{\text {loss }}\right)$ relates to the imaginary part of the complex effective index. $\mathrm{C}_{\text {loss }}$ is shown in the equation 4.

$$
C_{\text {loss }}=\frac{40 \pi}{\ln (10) \lambda} \operatorname{Im}\left(n_{\text {eff }}\right)\left[\frac{d B}{m}\right] \ldots \ldots \ldots \ldots \ldots \ldots \ldots \ldots \ldots \ldots . . . . . \cdots
$$

Where $\lambda$ is the operating wavelength. $I_{m}\left(n_{e f f}\right)$ is the imaginary value of the effective refractive index

\subsection{Analysis of the PCF}

The mode profile of the three photonic crystal fiber structures for $\mathrm{x}$ and $\mathrm{y}$ polarizations respectively at wavelength of $1550 \mathrm{~nm}$ are shown in Figures 1.4, 1.5 and 1.6. The results show that the mode is confined well in the core region of the PCF1 to PCF3 in $\mathrm{x}$ and $\mathrm{y}$ polarizations.

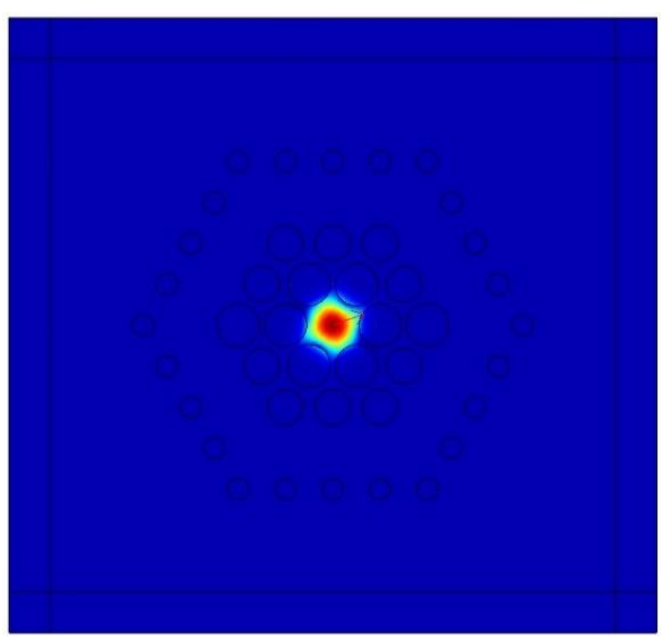

Figure 1.4a: Fundamental profile of PCF 1 at $1550 \mathrm{~nm}$ for $x$-polarization 


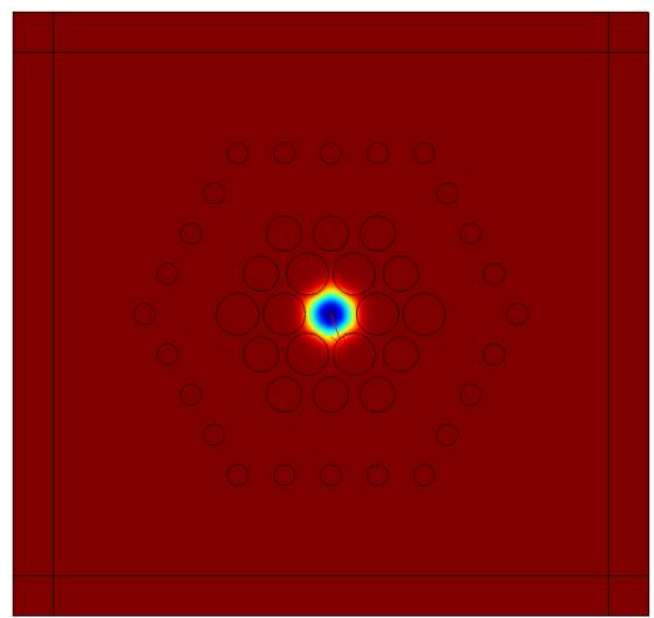

Figure 1.4b: Fundamental mode profile of PCF 1 at 1550nm for y-polarization

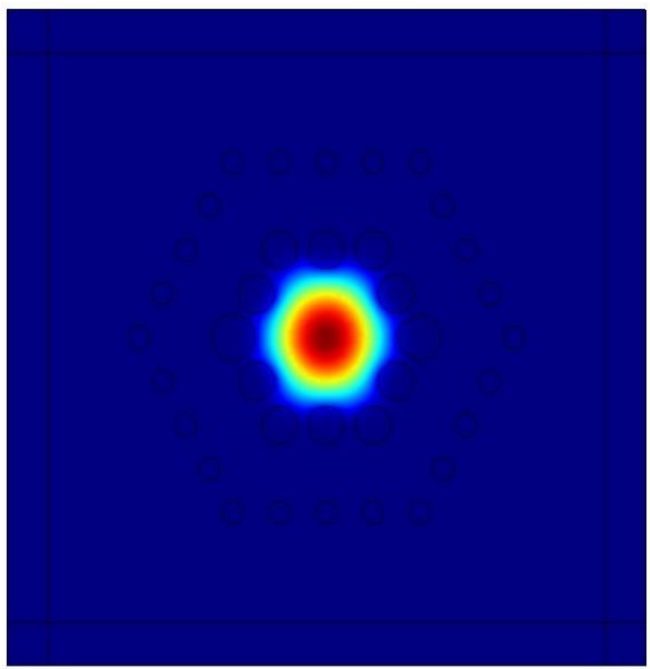

Figure 1.5a: Fundamental mode profile of PCF 2 at $1550 \mathrm{~nm}$ for $x$-polarization

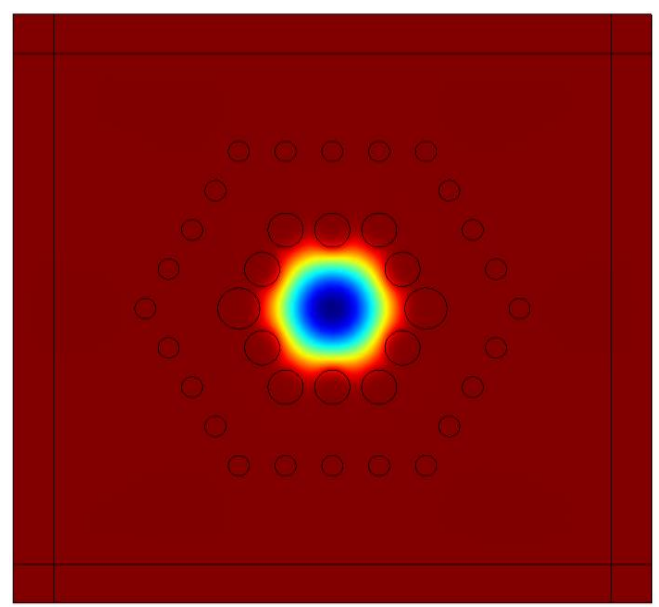

Figure 1.5b: Fundamental mode profile of PCF 2 at 1550nm for $y$-polarization 


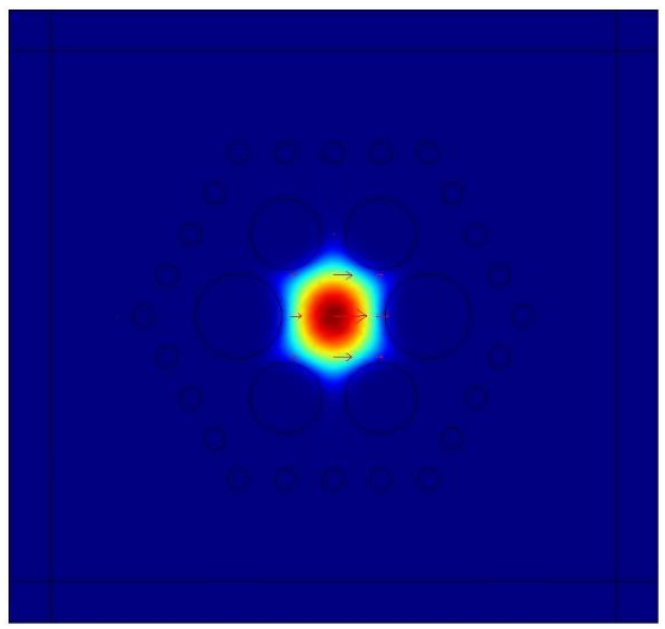

Figure 1.6a: Fundamental mode profile of PCF 3 at $1550 \mathrm{~nm}$ for $x$-polarization

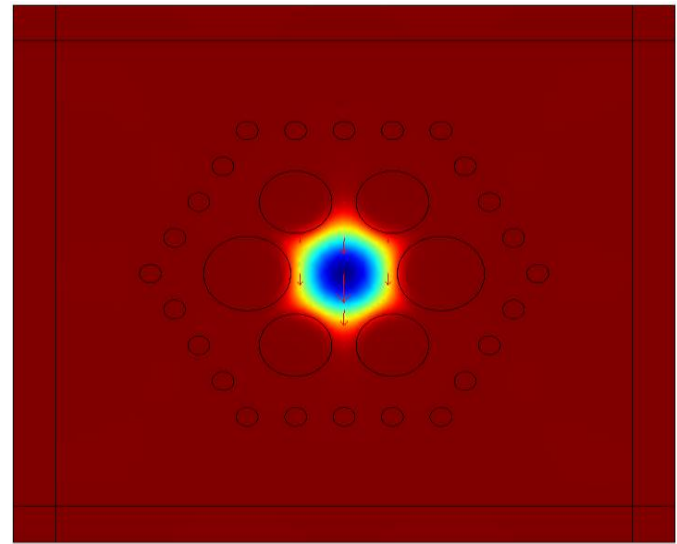

Figure 1.6b: Fundamental mode profile of PCF 3 at 1550 nm for y-polarization

\subsection{Results and Discussions}

\subsection{Change of wavelength with respect to Effective Refractive Index}

Effective refractive index values were obtained from the simulation. The variation of refractive index with wavelength is shown in Figure 1.7. The graph shows that as effective refractive index decreases, wavelength also increase, which is in agreement with (Akowuah, Ademgil, \& Haxha, 2012) for all the defects created.

The graph in figure 1.7 of effective refractive index indicates that PCF 1 has the lowest refractive index of $1.39 \mu \mathrm{m}$ at $1550 \mathrm{~nm}$ wavelength. The results in figure 1.7 show that light is confined well in core region at shorter wavelengths. Removing the third ring only to create defect, reduced the real effective refractive index as compared to the case where the first and second ring are removed. Also removing entirely, the first, second and third ring and replacing it with the single ring with larger holes also increased the real effective part of the complex wave. This shows that removing the inner rings makes room for increase in the real refractive index. 


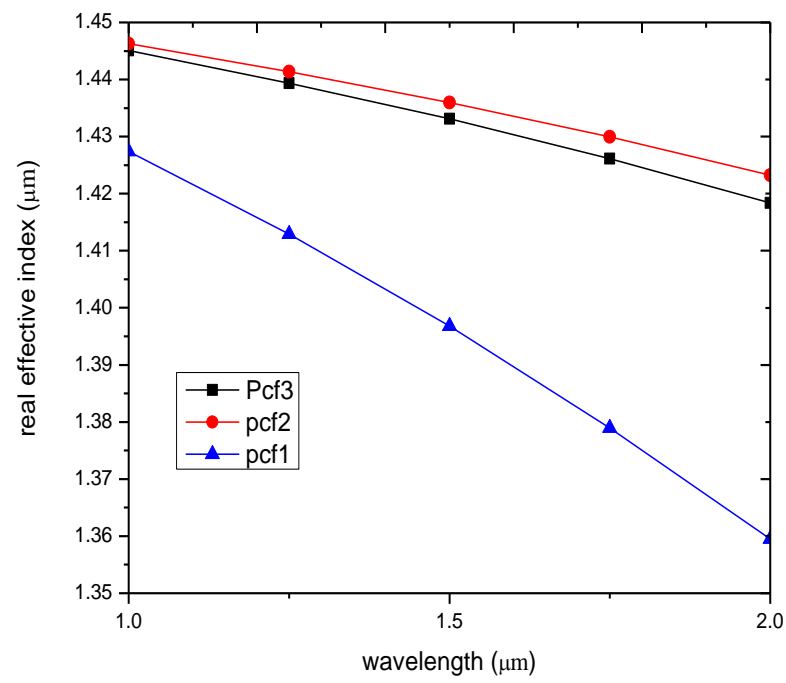

Figure 1.7: Real effective index of all three PCF designs

\subsection{Change of wavelength with respect to Chromatic dispersion}

The change of chromatic dispersion with wavelength for the proposed PCFs is shown in Figure 1.8 which shows that chromatic dispersion increases as wavelength increases. The dispersion of $89 \mathrm{ps} / \mathrm{km} . \mathrm{nm}$, around 100 ps/km.nm is obtained for PCFs 2 and 3 respectively and $176 \mathrm{ps} / \mathrm{nm} . \mathrm{km}$ for PCF 1 at $1550 \mathrm{~nm}$. The chromatic dispersion is better for PCF2 in which the first and third ring is removed. Minimum dispersion of $-140 \mathrm{ps} / \mathrm{km} . \mathrm{nm}$ for PCF 2, $-132 \mathrm{ps} / \mathrm{km} . \mathrm{nm}$ for PCF 3 and $-69 \mathrm{ps} / \mathrm{km} . \mathrm{nm}$ for PCF 1 is obtained at the value of $750 \mathrm{~nm}$. Zero chromatic dispersion has been obtained for all the proposed PCF structures from 0.85 to $1.1 \mathrm{ps} / \mathrm{km}$.nm which is also in agreement with (Barrientos-García et al., 2016; García et al., 2015).

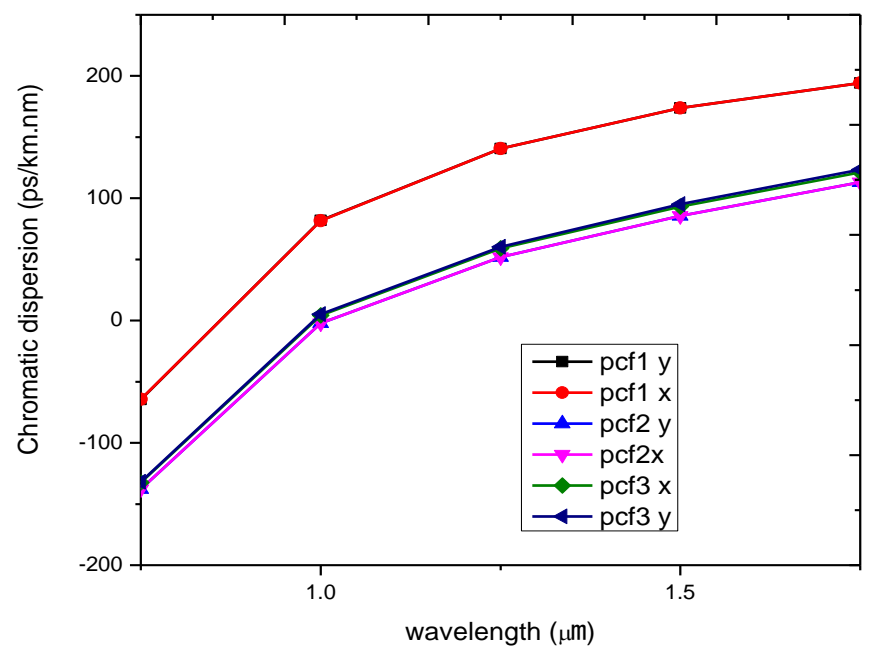

Figure 1.8: Chromatic Dispersion of all three PCF designs

\subsection{Change of wavelength with respect to $C_{\text {loss }}$}

The $\mathrm{C}_{\text {loss }}$ of PCF has been calculated using the imaginary part of the complex effective refractive index. Variation of $\mathrm{C}_{\text {loss }}$ with respect to wavelength is shown in Figure 1.9. The $\mathrm{C}_{\text {loss }}$ increases with increase in wavelength. The 
results show that PCF1 has the lowest confinement loss and this is attributed to the presence of more air holes than the rest of the PCFs since only the third ring is removed. The confinement loss in order of $10^{-2} \mathrm{~dB} / \mathrm{m}$ is obtained at the telecommunication wavelength of $1550 \mathrm{~nm}$ for PCFs 2 and 3, as shown in Figure 1.9. PCF 1 shows a significantly less confinement loss of less than $1.17 \times 10^{-4} \mathrm{~dB} / \mathrm{m}$ at $1550 \mathrm{~nm}$ which is better than (Hasan et al., 2017; Islam et al., 2019; Lee et al., 2016). It is worth noting that, considering the spectral range of $750 \mathrm{~nm}$ to $1500 \mathrm{~nm}$, confinement loss for all the designed PCFs, gradually increases as wavelength increases.

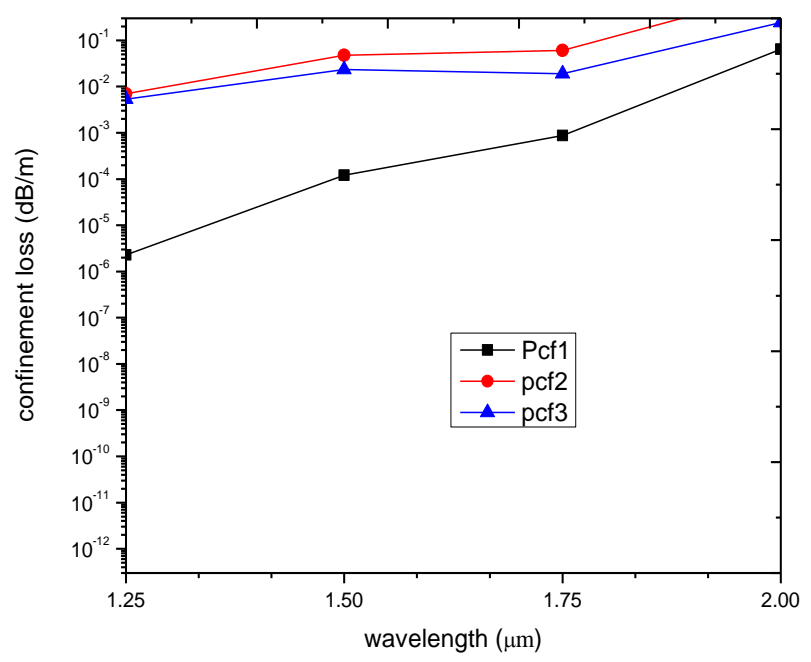

Figure 1.9: Confinement loss for PCF1, PCF2 and PCF3 designs

A key focus of the proposed design is to ensure the ease of fabrication. Some techniques which are being adopted for fabrication of PCFs are stack and draw method(Pysz et al., 2014) conventional drilling, (Ghosh, Bose, Roy, \& Bhadra, 2015), and sol-gel casting (Bise \& Trevor, 2005). The shape of the core along with the cladding is controlled utilizing stack and draw method and Sol-gel casting. It has been reported in (Liu, Wu, Vincent Tse, Lu, \& Tam, 2013) and (Bise \& Trevor, 2005) that complex structures of PCFs can be fabricated utilizing stack and draw methods and sol-gel techniques. Circular air holes, has been employed for the proposed designs and the number of rings, are also less bulky with the introduction of the defects. The proposed designs can be fabricated using stack and draw method.

\section{Conclusion}

It has been demonstrated that ring defects affect the chromatic dispersion and confinement loss. The proposed fibers show low $\mathrm{C}_{\text {loss }}$ and single zero chromatic dispersion for all the PCFs. The PCF 2 has given a negative chromatic dispersion of $-140 \mathrm{ps} / \mathrm{km} . \mathrm{nm}$ at $890 \mathrm{~nm}$ wavelength. The PCF1 shows very low confinement loss of $1.17 \times 10^{-4} \mathrm{~dB} / \mathrm{km}$ at $1.55 \mu \mathrm{m}$, chromatic dispersion of $-69 \mathrm{ps} / \mathrm{km} . \mathrm{nm}$ at $0.75 \mu \mathrm{m}$ and zero chromatic dispersion at $0.86 \mu \mathrm{m}$ which would be convenient for usage in short and medium transmission. 


\section{References}

Akowuah, E., Ademgil, H., \& Haxha, S. (2012). Design and analysis of photonic crystal fibres (PCFs) for broadband applications. Paper presented at the Adaptive Science \& Technology (ICAST), 2012 IEEE 4th International Conference on.

Barrientos-García, A., Sukhoivanov, I. A., Andrade-Lucio, J., Hernandez-Garcia, J., Ramos-Ortiz, G., IbarraManzano, O., \& Guryev, I. V. (2016). Numerical analysis of supercontinuum generation in photoniccrystal fibers with zero dispersion wavelengths in telecommunication windows. Optik, 127(22), 1098110990.

Bise, R. T., \& Trevor, D. J. (2005). Sol-gel derived microstructured fiber: Fabrication and characterization. Paper presented at the OFC/NFOEC Technical Digest. Optical Fiber Communication Conference., Anaheim.

Biswas, S. K., Arfin, R., Habib, A. B., Amir, S. B., Zahir, Z. B., Islam, M. R., \& Hussain, M. (2019). A Modified Design of a Hexagonal Circular Photonic Crystal Fiber with Large Negative Dispersion Properties and Ultrabigh Birefringence for Optical Broadband Communication. Paper presented at the Photonics.

Bjarklev, A., Broeng, J., \& Bjarklev, A. S. (2012). Photonic crystal fibres: Springer Science \& Business Media.

d ric Zolla, F. (2005). Foundations of photonic crystal fibres: Imperial College Press.

García, A. B., Sukhoivanov, I. A., Lucio, J. A. A., Manzano, O. G. I., Guryev, I., García, J. C. H., \& Ortiz, G. R. (2015). Numerical study of highly nonlinear photonic crystal fiber with tunable zero dispersion wavelengths. Journal of Electromagnetic Analysis and Applications, 7(05), 141.

Ghosh, D., Bose, S., Roy, S., \& Bhadra, S. K. (2015). Design and Fabrication of Microstructured Optical Fibers with Optimized Core Suspension for Enhanced Supercontinuum Generation. Journal of Lightwave Technology, 33.

Ghunawat, A. K., Chandra, R., \& Singh, G. (2017). Design of an ultra-flattened negative dispersion elliptical spiral photonic crystal fiber with high nonlinearity and high birefringence. Paper presented at the 2017 International Conference on Computer, Communications and Electronics (Comptelix).

Halder, A., \& Hossain, S. A. (2016). Design of ultra-high birefringent broadband dispersion compensating photonic crystal fiber for high speed transmission system. Imperial J. of Interdisc. Res, 2, 211.

Hasan, M. R., Islam, M. A., Rifat, A. A., \& Hasan, M. I. (2017). A single-mode highly birefringent dispersioncompensating photonic crystal fiber using hybrid cladding. journal of Modern Optics, 64(3), 218-225.

Islam, M. I., Ahmed, K., Paul, B. K., Chowdhury, S., Sen, S., Islam, M. S., . . . Bahar, A. N. (2019). Ultra-high negative dispersion and nonlinearity based single mode photonic crystal fiber: design and analysis. Journal of Optics, 48(1), 18-25.

Lee, Y. S., Lee, C. G., Jung, Y., Oh, M.-k., \& Kim, S. (2016). Highly birefringent and dispersion compensating photonic crystal fiber based on double line defect core. Journal of the Optical Society of Korea, 20(5), 567-574.

Liu, Z., Wu, C., Vincent Tse, M.-L., Lu, C., \& Tam, H.-Y. (2013). Ultrahigh birefringence index-guiding photonic crystal fiber and its application for pressure and temperature discrimination. Optiks Letter, 38.

Pysz, D., Kujawa, I., Stępień, R., Klimczak, M., Filipkowski, A., Franczyk, M., . . Buczyński, R. (2014). Stack and draw fabrication of soft glass microstructured fiber optics. Bulletin of the Polish Academy of Sciences. Technical Sciences, 62(4).

Talukder, H., Isti, M. I. A., Nuzhat, S., \& Biswas, S. K. (2020). Ultra-High Negative Dispersion Based Single Mode Highly Nonlinear Bored Core Photonic Crystal Fiber (HNL-BCPCF): Design and Numerical Analysis. Brazilian Journal of Physics, 1-9.

Yablonovitch, E. (1987). Inhibited spontaneous emission in solid-state physics and electronics. Physical review letters, 58(20), 2059. 